\title{
THE GROMOV INVARIANTS OF RUAN-TIAN AND TAUBES
}

\author{
Eleny-Nicoleta Ionel and Thomas H. Parker
}

C. Taubes has recently defined Gromov invariants for symplectic four-manifolds and related them to the Seiberg-Witten invariants ([T1], [T2]). Independently, Y. Ruan and G. Tian defined symplectic invariants based on ideas of Witten $([R T])$. While similar in spirit, these two sets of invariants are quite different in their details.

In this note we show that Taubes' Gromov invariants are equal to certain combinations of Ruan-Tian invariants (Theorem 4.5). This link allows us to generalize Taubes' invariants. For each closed symplectic four-manifold, we define a sequence of symplectic invariants $G r_{\delta}, \delta=0,1,2 \ldots$ The first of these, $G r_{0}$, generates Taubes' invariants, which count embedded $J$-holomorphic curves. The new invariants $G r_{\delta}$ count immersed curves with $\delta$ double points.

In particular, these results give an independent proof that Taubes' invariants are well-defined. Combined with Taubes' Theorem [T1], they also show that, for symplectic 4-manifolds with $b^{+}>1$, some of the Ruan-Tian symplectic invariants agree with the Seiberg-Witten invariants.

\section{Gromov invariants}

Fix a closed symplectic four-manifold $(X, \omega)$. Following the ideas of Gromov and Donaldson, one can define symplectic invariants by introducing an almost complex structure $J$ and counting (with orientation) the number of $J$ holomorphic curves on $X$ satisfying certain constraints. Unfortunately, technical difficulties make it necessary to modify the straightforward count in order to obtain an invariant. In this section we review the general construction and describe how the technicalities have led to two types of Gromov invariants.

Given $(X, \omega)$, one can always choose an almost complex structure $J$ tamed by $\omega$, i.e. with $\omega(Z, J Z)>0$ for all tangent vectors $Z$. A map $f: \Sigma \rightarrow X$ from a topological surface $\Sigma$ is called $J$-holomorphic if there is a complex structure $j$ on $\Sigma$ such that

$$
\bar{\partial}_{J} f=0
$$

where $\bar{\partial}_{J} f=\frac{1}{2}(d f \circ j-J \circ d f)$. The image of such a map is a $J$-holomorphic curve. Conversely, each immersed $J$-holomorphic curve is uniquely specified by

Received April 22, 1997.

The first author was partially supported by a M.S.R.I. Postdoctoral Fellowship

The second author was partially supported by N.S.F. grant DMS-9626245 
the equivalence class of a $J$-holomorphic pair $(f, j)$ under the action of the group of diffeomorphisms of $\Sigma$. These equivalence classes $[(f, j)]$ form moduli spaces

$$
\mathcal{M}_{A, g}
$$

labeled by the genus $g$ of $\Sigma$ and the class $A \in H_{2}(X)$ of the image (and implicitly depending on $J)$. The formal tangent space to $\mathcal{M}_{A, g}$ at $[(f, j)]$ can be identified with the kernel of the operator

$$
D_{f, j}: \Gamma\left(f^{*} N\right) \rightarrow \Omega^{0,1}\left(f^{*} N\right)
$$

obtained by linearizing (1.1) and restricting to the normal bundle $N$ along the image of $f$. The Riemann-Roch Theorem shows that

$$
\operatorname{dim} \mathcal{M}_{A, g}=2[g-1-\kappa \cdot A]
$$

where $\kappa$ is the canonical class of $(X, J)$. We can elaborate on this construction by marking $d$ points $x_{i}$ on $\Sigma$. The equivalence classes $\left[\left(f, j, x_{1}, \ldots, x_{d}\right)\right]$ of marked $J$-holomorphic curves then form a moduli space $\mathcal{M}_{A, g, d}$ of dimension $2[g-1-$ $\kappa \cdot A+d]$, and the evaluations $x_{i} \mapsto f\left(x_{i}\right)$ define a map

$$
\text { ev }: \mathcal{M}_{A, g, d} \rightarrow X^{d}=X \times \ldots \times X
$$

The marked points enable us to restrict attention to curves satisfying constraints. For our purposes it is almost always enough to consider point constraints. Thus we pick

$$
d=d_{A, g}=g-1-\kappa \cdot A
$$

generic points $p_{i}$ in $X$ and consider the constrained moduli space

$$
\mathcal{M}_{A, g}^{\prime}=e v^{-1}\left(p_{1}, \ldots, p_{d}\right)
$$

of all $J$-holomorphic curves that pass through the (ordered) points $p_{i}$. For generic $J$ and $\left\{p_{i}\right\}$, this constrained moduli space is zero-dimensional and its formal tangent space at $C=\left[\left(f, j, x_{1}, \ldots, x_{d}\right)\right]$ is the kernel of the restriction $D_{C}$ of (1.2) to the subspace of $\Gamma\left(f^{*} N\right)$ that vanishes at each marked point. Each curve $C \in \mathcal{M}_{A, g}^{\prime}$ therefore has a sign given by $(-1)^{S F}$ where SF denotes the spectral flow from $D_{C}$ to any complex operator $\bar{\partial}_{C}$ which is a compact perturbation of $D_{C}$. Counting the points in $\mathcal{M}_{A, g}^{\prime}$ with sign gives a "Gromov invariant"

$$
G r_{A, g}\left(p^{d}\right)=\sum_{C \in \mathcal{M}_{A, g}^{\prime}} \operatorname{sgn} C=\sum_{C \in \mathcal{M}_{A, g}^{\prime}}(-1)^{S F\left(D_{C}\right)} .
$$

One then tries to mimic Donaldson's cobordism arguments to show that $G r_{A, g}$ is independent of $J$ and $\left\{p_{i}\right\}$, and hence defines a symplectic invariant. This involves considerable analysis, and along the way one encounters a major technical difficulty $-\mathcal{M}_{A, g}$ may not be a manifold at the multiply-covered maps. There currently exist two distinct ways of overcoming this difficulty. 
(1) Taubes restricts $g$ to be the genus expected for embedded curves and counts embedded, not necessarily connected, $J$-holomorphic curves, dealing with the complications associated with multiply-covered curves. In the end he obtains 'Gromov-Taubes' invariants that we will denote by $G T_{0}(A)$.

(2) Ruan-Tian [RT] observed that the difficulties with multiply-covered maps can be overcome by replacing (1.1) by the inhomogeneous equation

$$
\bar{\partial}_{J} f=\nu
$$

where $\nu$ is an appropriate perturbation term. We will denote the resulting symplectic invariants by $R T(A, d)$.

The next two sections give some details about these two sets of invariants and describe generating functions involving them.

\section{The Taubes series}

The details of Taubes' construction are interesting and surprisingly subtle. Given $A \in H_{2}(X, \mathbb{Z})$, Taubes fixes the genus to be

$$
g_{A}=1+\frac{1}{2}(A \cdot A+\kappa \cdot A) .
$$

The moduli space of such curves has $\operatorname{dim} \mathcal{M}_{A}=A \cdot A-\kappa \cdot A$, so we constrain by $d_{A}=\frac{1}{2}(A \cdot A-\kappa \cdot A)$ points. The adjunction formula implies that each constrained curve is embedded unless $A$ lies in the set

$$
T=\left\{A \in H_{2}(X, \mathbb{Z}) \mid A^{2}=0 \text { and } \kappa \cdot A=0\right\},
$$

in which case the curve is a multiple cover of an embedded torus and $d_{A}=0$. Similarly, each constrained curve in

$$
\mathcal{E}=\left\{A \in H_{2}(X, \mathbb{Z}) \mid A^{2}=-1\right\},
$$

is an embedded "exceptional" sphere.

More generally, for each class $A$ and $d \geq 0$ we get a count of connected curves through $d$ generic points

$$
\operatorname{Gr}(A, d)
$$

defined by (1.3) with $g=d+1+\kappa \cdot A$. Note that by the adjunction formula

$$
d_{A}-d=g_{A}-g=\delta \geq 0
$$

so $0 \leq d \leq d_{A}$ with $d_{A}=0$ for $A \in \mathcal{E} \cup T$. Geometrically, $\delta$ is the number of double points on a generic immersed $A$-curve.

Taubes observed that for $A \in T, G r(A, 0)$ depends on $J$, as follows. For an embedded torus $C$, let $L_{i}, i=1,2,3$ be the three non-trivial real line bundles over $C$. Twisting the linearization $D_{C}$ by $L_{i}$ gives operators

$$
D_{i}: \Gamma\left(f^{*} N \otimes L_{i}\right) \rightarrow \Omega^{0,1}\left(f^{*} N \otimes L_{i}\right) .
$$


The space of almost complex structures is divided into chambers by the codimension one "walls" consisting of those $J$ for which there is a $J$-holomorphic curve with either $D_{C}$ or one of the $D_{i}$ not invertible. The value of $\operatorname{Gr}(A, 0)$ changes as $J$ crosses a wall.

Within a chamber, there are four types of $J$-holomorphic tori, labeled by the number $k=0,1,2,3$ of the $D_{i}$ whose sign (determined by the spectral flow) is negative. Thus for generic $J$, the moduli space of $J$-holomorphic $A$-curves is the disjoint union of four zero-dimensional moduli spaces $\mathcal{M}_{A, k}$. Counting with sign gives four "Taubes numbers"

$$
\tau(A, k)=\sum_{C \in \mathcal{M}_{A, k}} \operatorname{sgn} C .
$$

Taubes derived wall-crossing formulas and showed that a certain combination of the $\tau(A, k)$ is independent of $J$.

The right combination is best described by assembling the counts (2.4) and (2.5) into a single quantity associated with $X$. For that purpose, we introduce formal symbols $t_{A}$ for $A \in H_{2}(X ; \mathbb{Z})$ with relations $t_{A+B}=t_{A} t_{B}$ and specify three "generating functions" $e(t), f(t)$ and $g(t)$. From $f$ we construct functions $f_{k}$ corresponding to the four types of curves by setting

$$
f_{0}=f, \quad f_{1}(t)=\frac{f(t)}{f\left(t^{2}\right)}, \quad f_{2}(t)=\frac{f(t) f\left(t^{4}\right)}{f^{2}\left(t^{2}\right)}, \quad f_{3}(t)=\frac{f(t) f\left(t^{4}\right)}{f^{3}\left(t^{2}\right)} .
$$

in accordance to the wall crossing formulas in [T2]. We will also use another variable $s$ to keep track of the number of double points.

Definition 2.1. The Taubes Series of $(X, \omega)$ with generating functions $e, f$ and $g$ is the formal power series in the variables $t_{A}$ and $s$ defined by

$$
\begin{aligned}
& G T_{X}(t, s)= \\
& \prod_{E \in \mathcal{E}} e\left(t_{E}\right)^{G r(A, 0)} \cdot \prod_{A \notin T \cup \mathcal{E}} \prod_{d=0}^{d_{A}} g\left(t_{A} \frac{s^{d}}{d !}\right)^{G r(A, d)} \cdot \prod_{A \in T} \prod_{k=0}^{3} f_{k}\left(t_{A}\right)^{\tau(A, k)}
\end{aligned}
$$

with the $f_{k}$ given by $(2.6)$.

We then get a sequence of maps $G T_{\delta}: H_{2}(X ; \mathbb{Z}) \rightarrow \mathbb{Z}$ by expanding $(2.7)$ as a power series in $s$ :

$$
G T(t, s)=\sum_{A} \sum_{\delta=d_{A}-d} G T_{\delta}(A) t_{A} \frac{s^{d}}{d !}
$$

where we have labeled the coefficients by $\delta=d_{A}-d$ rather than $d$.

Proposition 2.2. With the choice

$$
e(t)=1+t, \quad f(t)=\frac{1}{1-t}, \quad \text { and } \quad g(t)=e^{t}
$$


the degree zero component $G T_{0}$ in (2.8) is the Gromov invariant defined by Taubes in [T2].

Proof. The coefficient $G T_{\delta}(A)$ of $t_{A} s^{d} / d$ ! in (2.7) is a sum of coefficients, one for each product of monomials $\left(t_{A_{i}} s^{d_{i}}\right)^{n_{i}}$ with $d=\sum n_{i} d_{i}$ and $A=\sum n_{i} A_{i}$, where the $A_{i}$ are distinct homology classes, $n_{i} \geq 0$, and $n_{i}=1$ for all $A_{i} \in \mathcal{E}$ (because the generating function is $e(t)=1+t$ ). Given such a decomposition, we can expand $\delta=d_{A}-d=d_{A}-\sum n_{i} d_{i}$ by writing $\delta_{i}=d_{A_{i}}-d_{i} \geq 0$ as in (2.4) and using the definition of $d_{A}$. This gives

$$
\begin{aligned}
\delta & =\frac{1}{2}\left[\left(\sum n_{i} A_{i}\right)^{2}-\sum n_{i} A_{i}^{2}\right]+\sum n_{i} \delta_{i} \\
& =\sum \frac{1}{2} n_{i}\left(n_{i}-1\right) A_{i}^{2}+\sum_{i<j} n_{i} n_{j} A_{i} A_{j}+\sum n_{i} \delta_{i}
\end{aligned}
$$

Each of the terms in this sum are nonnegative since (a) $A_{i}^{2} \geq 0$ for $A_{i} \notin \mathcal{E}$ and $n_{i}=1$ for $A_{i} \in \mathcal{E}$, and (b) $A_{i} \cdot A_{j} \geq 0$ for $i \neq j$ because the $A_{i}$ are distinct. Consequently, the only monomials that contribute to the $\delta=0$ term are those corresponding to decompositions of $A$ and $d$ with

(a) $n_{i}=1$ unless $A_{i}^{2}=0$,

(b) $A_{i} \cdot A_{j}=0$ for all $i \neq j$,

(c) $d_{i}=d_{A_{i}}$.

Let $\mathcal{S}=\mathcal{S}(A)$ be the set of such decompositions. For each $y=\left\{\left(n_{i}, A_{i}\right)\right\}$ in $\mathcal{S}$, let $y^{\prime}$ be the set of those $\left(n_{i}, A_{i}\right) \in y$ with $A_{i} \notin T$, let $y^{\prime \prime}$ be the set of those $\left(n_{i}, A_{i}\right) \in y$ with $A_{i}$ primitive and $A_{i} \in T$, and let $t_{y^{\prime}}$ and $t_{y^{\prime \prime}}$ be the corresponding monomials. Putting the functions (2.9) into (2.7), one sees that the coefficient of $t_{A} s^{d_{A}} / d_{A}$ ! has the form

$$
G r_{0}(A)=\sum_{y \in \mathcal{S}} R\left(y^{\prime}\right) Q\left(y^{\prime \prime}\right) .
$$

Here $R\left(y^{\prime}\right)$ is the coefficient of $t_{y^{\prime}} s^{d} / d$ ! in

$$
\prod_{A_{i} \notin T}\left[\exp \left(t_{A_{i}} \frac{s^{d_{i}}}{d_{i} !}\right)\right]^{\operatorname{Gr}\left(A_{i}, d_{i}\right)}
$$

(after noting that $t_{y^{\prime}}$ is at most linear in $t_{A_{i}}$ for each $A_{i} \in \mathcal{E}$ and $\exp t=$ $\left.1+t+O\left(t^{2}\right)\right)$, so

$$
R\left(y^{\prime}\right)=d ! \prod_{\left(n_{i}, A_{i}\right) \in y^{\prime}} \frac{G r\left(A_{i}, d_{i}\right)^{n_{i}}}{n_{i} !\left(d_{i} !\right)^{n_{i}}}
$$

Similarly, $Q\left(y^{\prime \prime}\right)$ is the coefficient of $t_{y^{\prime \prime}}$ in

$$
\prod_{A_{i} \in T} \prod_{k=0}^{3} f_{k}\left(t_{A_{i}}\right)^{\tau\left(A_{i}, k\right)}=\prod_{\substack{A_{i} \in T \\ \text { primitive }}} \prod_{q=1}^{\infty} \prod_{k=0}^{3} f_{k}\left(t_{A_{i}}^{q}\right)^{\tau\left(q A_{i}, k\right)}
$$


Then

$$
Q\left(y^{\prime \prime}\right)=\prod_{\left(n_{i}, A_{i}\right) \in y^{\prime \prime}} Q\left(n_{i}, A_{i}\right)
$$

where $Q(n, A)$ is the coefficient of $t_{A}^{n}$ in

$$
\prod_{q=1}^{\infty} \prod_{k=0}^{3} f_{k}\left(t_{q A}\right)^{\tau(q A, k)} .
$$

For each embedded, holomorphic torus $C$, let $f_{C}$ denote the function $f_{k}$ (resp. $1 / f_{k}$ ) when $C$ is of type $k$ and has positive (resp. negative) sign. Expanding $f_{C}(t)=\sum_{m} r(C, m) t^{m}$, we have

$$
Q(n, A)=\sum_{\mathcal{D}} \prod r\left(C_{j}, m_{j}\right)
$$

where $\mathcal{D}$ is the set of all pairs $\left(m_{j}, C_{j}\right)$ of $J$-holomorphic curves $C_{j}$ and multiplicities $m_{j}$ with $\left[C_{j}\right]=q_{j} A$ and $\sum m_{j} q_{j}=n$. Together, (2.10) - (2.13) exactly agree with the invariant defined by Taubes ([T2] section $5 \mathrm{~d}$ ).

Remark 2.3. Taubes chooses the functions (2.9) to make his invariants agree with the Seiberg-Witten invariants.

The numbers $G r_{\delta}(A)$ defined by (2.7) and (2.8) count the $J$-holomorphic $A$ curves (of any genus and any number of components) with $\delta$ double points, and thus generalize Taubes' count of embedded curves. Below, we will verify that the $G r_{\delta}(A)$ are symplectic invariants by relating the Taubes Series to Ruan-Tian invariants.

\section{The RT series}

Ruan and Tian [RT] define symplectic invariants $R T_{A, g, d}\left(\alpha_{1}, \ldots, \alpha_{d}\right)$ by taking the moduli space $\mathcal{M}_{A, g, d}$ of connected, perturbed holomorphic $A$-curves with genus $g$ and $d$ marked points, restricting to the subset $\mathcal{M}_{A, g, d}^{\prime}$ where the marked points lie on fixed constraint surfaces representing the $\alpha_{i} \in H_{*}(X)$, and counting with orientation (assuming $\mathcal{M}_{A, g, d}^{\prime}$ is zero-dimensional). In particular, when the $\alpha_{i}$ are all points and $g=d+1+\kappa \cdot A$ we get invariants

$$
R T(A, d)=R T_{A, d+1+\kappa \cdot A, d}\left(p^{d}\right) .
$$

This section describes how to assemble these invariants into a series analogous to $(2.7)$.

First we must deal with a technical problem. In [RT], the invariants $R T_{A, g, d}$ are defined only for the "stable range" $2 g+d \geq 3$. This leaves $R T(A, d)$ undefined for two types of curves: tori with no marked points, which occur when $d=$ $\kappa \cdot A=0$, and spheres with fewer than three marked points, which occur when $d=0,1,2$ and $d+1=-\kappa \cdot A$. But we can extend definition (3.1) to these cases 
by imposing additional "constraints" which are automatically satisfied. For this, choose a class $\beta \in H_{2}(X)$ with $A \cdot \beta \neq 0$ and set

$$
R T(A, 0)=\frac{1}{A \cdot \beta} R T_{A, 1,1}(\beta) \quad \text { if } \kappa \cdot A=0
$$

and

$$
R T(A, d)=\frac{1}{(A \cdot \beta)^{3-d}} R T_{A, 0,3}\left(p^{d} \beta^{3-d}\right) \quad \text { if } \quad d=\kappa \cdot A-1=0,1,2 .
$$

Thus defined, these invariants count perturbed holomorphic curves. For example, when $\kappa \cdot A=0$ each genus one curve $C$ (without marked points) representing $A$ is a map $f: T^{2} \rightarrow X$, well-defined up the automorphisms of $T^{2}$ with the induced complex structure. Fix a point $p \in T^{2}$ and represent $\beta$ by a cycle in general position. Then $C \cap \beta$ consists of $A \cdot \beta$ distinct points. Hence $C$ is the image of exactly $A \cdot \beta$ maps $f: T^{2} \rightarrow X$ with $f(p) \in \beta$ and these are counted by $R T_{A, 1,1}(\beta)$.

Now fix a generating function $F_{A}$ for each class $A$ and assign a factor $F_{A}\left(t_{A}\right)$ to each curve that contributes +1 to the count $R T(A, d)$, and a factor $1 / F_{A}\left(t_{A}\right)$ to each curve that contributes -1 . Taking the product gives a series in the variables $t_{A}$

$$
\prod_{A \in H_{2}(X)} F_{A}\left(t_{A}\right)^{R T(A, d)}
$$

which is an invariant of the deformation class of the symplectic structure of $(X, \omega)$. As with the Taubes Series, different choices of the $F_{A}$ give different series, but all encode the same data. We will choose three generating functions and form a series resembling (2.7).

Definition 3.1. The Ruan-Tian Series of $(X, \omega)$ defined by $e(t), F(t)$ and $g(t)$ is

$$
\begin{aligned}
& R T_{X}(t, s)= \\
& \prod_{E \in \mathcal{E}} e\left(t_{E}\right)^{R T(A, 0)} \cdot \prod_{A \notin T \cup \mathcal{E}} g\left(t_{A} \frac{s^{d}}{d !}\right)^{R T(A, d)} \cdot \prod_{A \in T} F\left(t_{A}\right)^{R T(A, 0)}
\end{aligned}
$$

Expanding in power series as in $(2.8)$ gives invariants $R T_{\delta}: H_{2}(X ; \mathbb{Z}) \rightarrow \mathbb{Z}$.

To make this more concrete, we could take $e(t), F(t)$ and $g(t)$ to be the specific functions given in (2.9). That choice, however, overcounts tori with self-intersection zero. It turns out that the formulas are simpler if $F$ satisfies

$$
\prod_{k=1}^{\infty} F\left(t^{k}\right)=e^{t}
$$


Thus it is appropriate to make the more awkward-looking choice

$$
e(t)=1+t, \quad F(t)=\exp \left(\sum_{m=1}^{\infty} \mu(m) t^{m}\right), \quad \text { and } \quad g(t)=e^{t}
$$

where $\mu$ is the Möbius function. (The Möbius function is defined by $\mu(1)=1$, $\mu(m)=(-1)^{k}$ if $m$ is a product of $k$ distinct primes, and $\mu=0$ otherwise.) One can then verify (3.4) by writing $\ell=m k$ and using the basic fact that

$$
\sum_{m \mid \ell} \mu(m)= \begin{cases}1 & \text { if } \ell=1 \\ 0 & \text { otherwise. }\end{cases}
$$

We will see next how the generating functions (3.5) lead back to the Taubes Series and the Seiberg-Witten invariants.

\section{Equivalence of the invariants}

In this section we will prove that the Taubes and Ruan-Tian Series are equal for any closed symplectic four-manifold. The proof is straightforward for classes $A \notin T$, but for the toroidal classes $A \in T$ it requires some combinatorics.

For classes $A \notin T$, the moduli space of $J$-holomorphic curves of genus $g_{A}$ passing through $d$ points contains no multiply covered curves for generic $J$ (cf. $[\mathrm{R}]$, [T2]). Consequently, the moduli space of such curves is smooth and the linearized operator has no cokernel. The Implicit Function Theorem then implies that each of these curves (but none of their multiple covers) can be uniquely perturbed to a solution of the equation $\bar{\partial}_{j} f=\nu$ for small $\nu$. Thus

$$
\operatorname{Gr}(A, d)=R T(A, d) \quad \text { for } \quad A \notin T,
$$

so the first two factors in the products (2.7) and (3.3) are equal.

The computations for $A \in T$ are more complicated because multiple covers $d o$ contribute. In this case, the moduli space $\mathcal{M}_{A}$ of $J$-holomorphic, connected, embedded $A$-curves is finite for generic $J$, and each curve $C \in \mathcal{M}_{A}$ is a torus. The last part of the Gromov series (3.3) has the form

$$
G r^{T}=\prod_{A \in T} \prod_{C \in \mathcal{M}_{A}} \phi_{C}\left(t_{A}\right)
$$

for some function $\phi_{C}$ that we must determine.

To do that, we fix one torus $C \in \mathcal{M}_{A}$ defined by an embedding $\left(T^{2}, x_{0}, j_{0}\right) \rightarrow$ $X$ and regard the domain $\left(T^{2}, x_{0}, j_{0}\right)$ as the quotient of the complex plane by the lattice

$$
\Lambda_{0}=\mathbb{Z} \oplus \tau \mathbb{Z}
$$

Curves $C^{\prime}$ which are $m$-fold covers of $C$ are given by pairs $(\psi, j)$ where $\psi$ : $\left(T^{2}, x_{0}, j\right) \rightarrow\left(T^{2}, x_{0}, j_{0}\right)$ is an $m$-fold cover map; these are classified (up to diffeomorphisms of the domain) by index $m$ sublattices $\Lambda \subset \Lambda_{0}$. Let $\mathcal{L}_{m}$ be the set of all such lattices. 
For generic $J$ the linearized operator has zero cokernel (it is invertible with index zero). Hence each $m$-fold cover can be uniquely perturbed to a solution of $\bar{\partial}_{j} f=\nu$, which contributes to $R T_{m A, 1,1}$. The total contribution of the multiple covers of $C$ to $R T_{m A, 1,1}$ is

$$
\sum_{\Lambda \in \mathcal{L}_{m}} \operatorname{sgn} \Lambda
$$

where $\operatorname{sgn} \Lambda$ is the sign of the multiple cover $C^{\prime}$ described by $\Lambda$. Thus, after stabilizing as in (3.2),

$$
\phi_{C}\left(t_{A}\right)=\prod_{m=1}^{\infty} F\left(t_{A}^{m}\right)^{\frac{1}{m}} \sum_{\Lambda \in \mathcal{L}_{m}} \operatorname{sgn} \Lambda
$$

To proceed, we must determine sgn $\Lambda$ using the orientation prescribed by Ruan-Tian. As in Section 1, this is given by the the spectral flow of the linearization $D_{C}$ (the exposition in [RT] is obscure, but this is clearly the orientation that the authors intended to specify). This sign is independent of $\nu$ for small $\nu$, so we can assume that $\nu=0$ in the subsequent calculations.

Lemma 4.1. The sign of a curve $C^{\prime}=\mathbb{C} / \Lambda$ is

$$
\operatorname{sgn} \Lambda=\operatorname{sgn} D_{0} \prod \operatorname{sgn} D_{i}
$$

where the product is over all $i=1,2,3$ such that $\Lambda_{0}$ is a sublattice of $\Lambda_{i}$ with $\Lambda_{i}$ defined by (4.5).

Proof. Looking at the explicit formula for $D_{C^{\prime}}$ [T2], one sees that $D_{C^{\prime}}$ is the pullback of $D_{C}$ (it depends only on the 1-jet of $J$ along $C$ ). Fix a complex operator $\bar{\partial}$ on $C$, choose a path from $\bar{\partial}$ to $D_{C}$, and let $D_{t}$ be the lifted path of operators on $C^{\prime}$; each $D_{t}$ is invariant under deck transformations. As in [T2], we can assume that $\operatorname{ker} D_{t}=\{0\}$ except at finitely many values of $t=t_{k}$, where ker $D_{t}$ is one-dimensional.

The translations of $\mathbb{C}$ by 1 and $\tau$ respectively induce deck transformations $\tau_{1}$ and $\tau_{2}$ of $C^{\prime} \rightarrow C$; these generate the abelian group $G=\Lambda_{0} / \Lambda$ of all deck transformations. At each $t=t_{k}$, ker $D_{t}$ is a one-dimensional representation $\rho_{i}$ of $G$, so is one of four possibilities:

$$
\left\{\begin{array} { l } 
{ \rho _ { 0 } \tau _ { 1 } ( \xi ) = \xi } \\
{ \rho _ { 0 } \tau _ { 2 } ( \xi ) = \xi }
\end{array} \quad \left\{\begin{array} { l } 
{ \rho _ { 1 } \tau _ { 1 } ( \xi ) = - \xi } \\
{ \rho _ { 1 } \tau _ { 2 } ( \xi ) = \xi }
\end{array} \quad \left\{\begin{array} { l } 
{ \rho _ { 2 } \tau _ { 1 } ( \xi ) = \xi } \\
{ \rho _ { 2 } \tau _ { 2 } ( \xi ) = - \xi }
\end{array} \quad \left\{\begin{array}{l}
\rho_{3} \tau_{1}(\xi)=-\xi \\
\rho_{3} \tau_{2}(\xi)=-\xi
\end{array}\right.\right.\right.\right.
$$

where $\xi$ is a generator of the kernel. Call these kernels of type $0,1,2$ and 3 respectively. Then

$$
S F=\sum_{i=0}^{3} S F_{i}
$$

where $\mathrm{SF}_{i}$ is the number of $t_{k}$ of type $i$ (counted with orientation), and

$$
\operatorname{sgn} \Lambda=(-1)^{\mathrm{SF}}=\prod(-1)^{\mathrm{SF}_{i}} .
$$


Note that each $\xi$ of type 0 descends to a section of ker $D_{t}$ on $\mathbb{C} / \Lambda_{0}$. In fact, this is a one-to-one correspondence, so $S F_{0}$ is the spectral flow of the path $D_{t}$ on the base curve $C$ and $(-1)^{\mathrm{SF}}$ is the sign of $D_{0}$. The remaining representations determine three index two sublattices

$$
\Lambda_{i}=\operatorname{ker} \rho_{i}
$$

of $\Lambda$. Thinking of $\xi$ as a $\Lambda$-invariant section on $\mathbb{C}$, one sees that (a) a type $i$ kernel cannot appear unless $\Lambda \subset \Lambda_{i}$, and (b) if $\Lambda \subset \Lambda_{i}$ then $\xi$ descends to an element of ker $D_{t}$ over the double cover $\mathbb{C} / \Lambda_{i}$. Thus $\mathrm{SF}_{i}$ vanishes if $\Lambda$ is not a subset of $\Lambda_{i}$, and when $\Lambda \subset \Lambda_{i} \mathrm{SF}_{i}$ coincides with the spectral flow of Taubes' operator $D_{i}$. Then (4.4) is the same as (4.3).

Remark 4.2. A set of representatives of the lattices in $\mathcal{L}_{m}$ is

$$
\Lambda=a \mathbb{Z}+(b \tau+p) \mathbb{Z} \quad \text { where } m=a b, \quad p=0, \ldots, a-1 .
$$

The group of deck transformations is $G \cong \mathbb{Z}_{a} \times \mathbb{Z}_{b}$, and the three the lattices (4.5) are

$$
\Lambda_{1}=\mathbb{Z}+2 \tau \mathbb{Z}, \quad \Lambda_{2}=2 \mathbb{Z}+\tau \mathbb{Z}, \quad \Lambda_{3}=2 \mathbb{Z}+(1+\tau) \mathbb{Z} .
$$

Now fix $m$ and separate the set of lattices $\mathcal{L}$ into:

$$
\begin{aligned}
\mathcal{L}^{0} & =\left\{\Lambda \in \mathcal{L} \mid \Lambda \text { is contained in none of the lattices } \Lambda_{1}, \Lambda_{2}, \Lambda_{3}\right\} \\
\mathcal{L}^{i} & =\left\{\Lambda \in \mathcal{L} \mid \Lambda \text { is contained in } \Lambda_{k} \text { only for } k=i\right\} \\
\mathcal{L}^{123} & =\left\{\Lambda \in \mathcal{L} \mid \Lambda \text { is contained in } \Lambda_{1}, \Lambda_{2} \text { and } \Lambda_{3}\right\}
\end{aligned}
$$

Note that if $\Lambda$ is contained in two of the $\Lambda_{i}$ then it is contained in the third. Thus the above sets constitute a partition

$$
\mathcal{L}=\mathcal{L}^{0} \cup \mathcal{L}^{1} \cup \mathcal{L}^{2} \cup \mathcal{L}^{3} \cup \mathcal{L}^{123}
$$

Furthermore, there are automorphisms of $\Lambda_{0}$ that interchange the lattices $\Lambda_{i}, i=$ $1,2,3$, so the sets $\mathcal{L}^{1}, \mathcal{L}^{2}$, and $\mathcal{L}^{3}$ have the same cardinality. Hence from (4.3) we have

$$
\sum_{\Lambda \in \mathcal{L}_{m}} \operatorname{sgn} \Lambda=\operatorname{sgn} D_{0}\left\{A+B \sum_{i=1}^{3} \operatorname{sgn} D_{i}+C \prod_{i=1}^{3} \operatorname{sgn} D_{i}\right\}
$$

where $A=\left|\mathcal{L}^{0}\right|$ is the number of elements of $\mathcal{L}^{0}, B=\left|\mathcal{L}^{1}\right|$, and $C=\left|\mathcal{L}^{123}\right|$.

Lemma 4.3. Set $\sigma(m)=\sum_{a \mid m} a$ if $m$ is a positive integer, and $\sigma=0$ otherwise. Then

$$
A+3 B+C=\sigma(m), \quad B+C=\sigma(m / 2), \quad C=\sigma(m / 4) .
$$


Proof. Using the representatives $(4.6)$ of $\mathcal{L}$, we have

$$
A+3 B+C=|\mathcal{L}|=\sum_{m=a b} \sum_{p=0}^{a-1} 1=\sum_{a \mid m} a=\sigma(m) .
$$

Next, $B+C$ is the number of lattices $\Lambda \in \mathcal{L}$ which contain $\Lambda_{1}$. These are the lattices (4.6) with $b=2 \beta$ even, so

$$
B+C=\sum_{m=a \cdot 2 \beta} \sum_{p=0}^{a-1} 1=\sum_{a \mid \frac{m}{2}} a=\sigma\left(\frac{m}{2}\right) .
$$

Finally, $\mathcal{L}^{123}$ is the set of all lattices $\Lambda$ such that $a, b$ and $p$ are all even. Writing $a=2 \alpha, b=2 \beta$, and $p=2 q$, we obtain

$$
C=\left|\mathcal{L}^{123}\right|=\sum_{m=4 \alpha \beta} \sum_{0 \leq 2 q \leq 2 \alpha-1} 1=\sum_{\alpha \mid \frac{m}{4}} \alpha=\sigma\left(\frac{m}{4}\right) .
$$

Proposition 4.4. The generating function $\phi_{C}$ of an embedded torus $C$ is

$$
\phi_{C}(t)=\left[f(t) f\left(t^{2}\right)^{s_{1} / 2} f\left(t^{4}\right)^{s_{2} / 4}\right]^{\operatorname{sgn} C}
$$

where

$$
s_{1}=\sum_{i=1}^{3} \operatorname{sgn} D_{i}-3, \quad s_{2}=\prod_{i=1}^{3} \operatorname{sgn} D_{i}-\sum_{i=1}^{3} \operatorname{sgn} D_{i}+2,
$$

and

$$
f(t)=\prod_{m \geq 1} F\left(t^{m}\right)^{\sigma(m) / m}
$$

Proof. From equations (4.2) and (4.7) and Lemma 4.3, we obtain

$$
\log \phi_{C}\left(t_{A}\right)=\operatorname{sgn} D_{0} \sum_{m=1}^{\infty} \frac{1}{m}\left[\sigma(m)+s_{1} \cdot \sigma\left(\frac{m}{2}\right)+s_{2} \cdot \sigma\left(\frac{m}{4}\right)\right] \log F\left(t_{A}^{m}\right)
$$

After substituting in (4.10), this gives (4.8).

When $C$ has Taubes' type 0 all three $D_{i}$ have positive sign, so $s_{1}=s_{2}=0$ in (4.9). Similarly, $\left(s_{1}, s_{2}\right)$ is $(-2,0)$ for type $1,(-4,4)$ for type 2 , and $(-6,4)$ for type 3 . Thus (4.8) gives

$$
\prod_{A \in T} \prod_{C \in \mathcal{M}_{A}} \phi_{C}\left(t_{A}\right)=\prod_{A \in T} \prod_{k=0}^{3} f_{k}\left(t_{A}\right)^{\tau(A, k)}
$$


where $f_{0}=f$ and

$$
f_{1}(t)=\frac{f(t)}{f\left(t^{2}\right)}, \quad f_{2}(t)=\frac{f(t) f\left(t^{4}\right)}{f^{2}\left(t^{2}\right)}, \quad f_{3}(t)=\frac{f(t) f\left(t^{4}\right)}{f^{3}\left(t^{2}\right)}
$$

- exactly as in (2.6)! Since the first factors in (2.7) and (3.3) are equal by (4.1), we have the following equivalence.

Theorem 4.5. For any closed symplectic four-manifold $(X, \omega)$, the Taubes and Ruan-Tian Series (2.7) and (3.3) coincide when $f$ and $F$ are related by (4.10):

$$
G T_{X}(t, s)=R T_{X}(t, s) .
$$

Hence Taubes' Gromov invariants $G T_{\delta}(A)$ depend only of the deformation class of $\omega$ and are computable from the Ruan-Tian invariants.

If we use the particular form of $F$ satisfying (3.4) and make the change of variable $m=a b$, we obtain

$\log f(t)=\sum_{m=1}^{\infty} \sum_{a \mid m} \frac{a}{m} \log F\left(t^{m}\right)=\sum_{b=1}^{\infty} \frac{1}{b} \sum_{a=1}^{\infty} \log F\left(t^{a b}\right)=\sum_{b=1}^{\infty} \frac{1}{b} t^{b}=\log \frac{1}{1-t}$.

Thus the Ruan-Tian Series with the generating functions defined in (3.5) exactly reproduces the Taubes Series with his choice of generating functions (2.9).

\section{References}

[R] Y. Ruan, Symplectic topology and complex surfaces, in Geometry and Topology of Complex Manifolds, T. Mabuchi, J. Noguchi and T. Ochial, eds. World Scientific Public., Singapore, 1994.

[RT] Y. Ruan and G. Tian, Higher genus symplectic invariants and sigma model coupled with gravity, preprint, 1996.

[T1] C. H. Taubes, From the Seiberg-Witten equations to pseudo-holomorphic curves, preprint, 1995.

[T2] - Counting pseudo-holomorphic curves in dimension 4, preprint, 1996.

MIT, CAmbridge, MA 02139

E-mail address: ionel@math.mit.edu

Michigan State University, East Lansing, MI 48824

E-mail address: parker@math.msu.edu 